\title{
Research on Digital Resources Integration Model in Cloud Computing Environment
}

Ying-jiang HAN

Henan Technical College of Construction

Zhengzhou City Zip Code: 450064

China

ABSTRACT: This article analyzed the present situation of digital resources integration, expounded the content and methods of the digital resources integration, focused on related digital resources integration theory for cloud computing environment, combined with the characteristics of cloud computing, and proposed the application and improvement of the digital resources integration based on cloud computing technology.

Keywords: Cloud Computing, Digital Resources, Resources Integration

Received: 8 May 2019, Revised 1 July 2019, Accepted 9 July 2019

DOI: $10.6025 /$ jisr $/ 2019 / 10 / 3 / 92-96$

\section{Introduction}

Before the arising of digital carrier, paper carrier was as the information carrier, supplemented by miniature carrier, laser carrier and carrier magnetic carrier such as a variety of compatible with each other and form a stable structure. Along with the formation of the digital and network environment, digital information has gradually become the mainstream media, information transmission and digital resources organization also became a main research direction in the field of information management and the work content.

According to IDC, also named International Data Corporation. In March 2013, the latest digital universal research report named "Big Data, larger number of figure, the largest growth was in the far east [1]". As the universal application of personal computers, smart phones and other devices and ever growing internet traffic in emerging market, It made the digital universe doubled its quantity within the last two years, an astounding 2.8ZB. IDC predicted that the digital universe would develop with the double the speed every year. Each person would have the possession of 5,247 GB of data on earth, and the digital universe scale would reach $40 \mathrm{ZB}$ till 2020.

Rapid development of digital information, the popularization of $3 \mathrm{G}$ and the development of wireless network, had inspired the enthusiasm of using digital information resources through the network. China Internet network information center (CNNIC) issued its $34^{\text {th }}$ report China internet network development state statistic report [2] on July 21, 2014. Up to June 2014, there were 632 million internet users, and Internet penetration rate was $46.9 \%$. Internet development focus shifted from "extensive" to 
“depth” transformation, various network application profoundly changed Internet life.

The above information indicated that the explosive growth of the digital universe had became the mainstream of the information resources and a variety of heterogeneous digital information. Digital resources presented a partial order, but the overall state was disorder. The application and popularity of cloud computing technology and intelligent terminal had fundamental change of behavior and method to obtain knowledge. The network, especially in wireless networks became the important channel for people to seek for information and knowledge. But digital resource isolated island and digital resources overloads had became the two serious factors to hinder the application of effective digital resources. Cloud computing technology brought new opportunity to digital resources. How to make full use of cloud computing technology to improve the use efficiency of digital resources. To effectively integrate and reorganize the large number of heterogeneous digital resources were the serious problem to solve the current digital resources integration process.

\section{Digital Resources and Digital Resources Integration}

Digital information had become the mainstream of information transferring media, Information resources had a fundamental change ether from the production, storage, or transport and use of digital resources organization. The digital resources organization had become the main research direction and work content in the field of information management.

\subsection{Digital Resources and Features}

Digital resources was information resources in the form of digital distribution, processing, through the network access, transfer and the sum of the development and utilization of information.

In the form of digital distribution, processing, through the network access, transfer and the sum of the development and utilization of information resources is known as digital resources, digital resources includes both traditional information resources digitization, also included the direct production of digital information in digital way. The digital information resources was also the information integration, ordering and facilitation, including text, charts, graphs, images, audio, video, etc all digital media literature. In the network environment, only the digital information could be the available information for users, the network environment was digital information production, dissemination and use of the main occasions. Digital resources was way of production, distribution, access, transfer and utilization of literature was totally different with traditional carrier, it had a high degree of sharing and partial order, the diversity of types and main characteristics such as dynamic resources. These characteristics of its organization and integration of digital information put forward the higher request, as the method and technology in digital resources integration and significant changes had taken place in the management model.

\subsection{Digital Resources Integration}

Digital resources integration was based on user needs as the guidance, based on the property characteristics of digital resources, according to certain principles and methods. Using the integration technology gathered scattered disorderly, relatively independent heterogeneous resources system integration, and through the technology and methods of knowledge organization, would be the integration of various information resources type, specification and scientifically integration and reorganization, and made its reorganizing a new organic whole, to form a better, more efficient new system of digital resources, in order to offer convenient, fast and efficient [3].

In a cloud environment of digital resource integration would not only concentrated the distributed heterogeneous resources, more important is to the content of the digital resources features and external for ordering and reveal, at the same time, description of the relationship between the digital resources would automate clustering and classification of digital resources and convenient user get on-demand, easy extension information and resources. Secondly, through the virtualization technology integration, a variety of digital resources integration, and the transition of disordered information flow becoming to the orderly flow of information, made the formation of more advanced information products and promote the development process each link of sharing resources, complemented each other, together to create value. Finally, for the integration of information resources indexing information resource cloud formation has greatly helped knowledge service model in digital information resources indexing the transparent interface to provide users with on-demand access to information resources of knowledge service.

\subsection{Basic Content of Digital Resources Integration}

From different fields of the study concept of digital resource integration, and based on the research field of digital resources integration of the scholars were also different. In a broad sense, the digital resources integration contented the digital resources 
optimization selection, analysis, describe and reveal, sorting and storage. The basic preparation of resources integration was selection and analysis of digital information, and its central content was the digital information resources described and reveal and orderly, Preparation is the basis of resource integration selection and analysis of digital information, is the central content of digital information resources is described and reveal and ordering. And several links of former formed orderly collection of information storage, represented the basic completion of the process of digital resources integration.

\subsubsection{Optimal Selection of Digital Resources}

Optimal selection was the first step of digital resources integration, and was found in the vast network of digital information ocean and been confirmed to have the function of organizing, sorting out and saving the value information. Due to the digital resources was easy to generate, distribute, reproduce and transmit, in the information generation stage, and the lack of a perfect choice, evaluation system, so the quality and value of digital information was uneven. Therefore, to integrate the digital information could improve the quality of information resources and optimizing choice.

\subsubsection{The Analysis of the Digital Resources}

Analysis of digital resources was according to certain logical relations, from the semantics, pragmatics and grammar for selection of digital resources, processing and finishing, through calculation, analysis, comparison research, It could create more and more resources and could reveal essence content of activity. It was the premise and foundation of digital resource description and reveal, and one of the indispensable important part of digital resources integration, has a direct impact on the quality of the digital resources integration has a direct impact. Through the analysis and research of digital resources, digital resources had more valuable to use and practical significance.

\subsubsection{The Description of the Digital Resources and Revealed}

Information description and reveal referred to the needs of digital resource integration and information retrieval, the information of the subject content, the form characteristics, such as morphological analysis, selection, record the activities. Information describing was mainly for the description of external characteristic; The content of the information disclosing information revealed the main characteristics. Information description and reveals were the important content of information organization, played an important role in information organization.

\subsubsection{Collection and Storage of Digital Resources}

Storage of digital resources was arranging the sequence of information and could be processed according to certain order format and stored in a specific carrier, its purpose was to managers and users of digital resources quickly and accurately identify, locate and retrieve information, cd-rom retrieval system, network information retrieval tools was the manner in which information stored. When the information was stored in various retrieval tools meant the end of the process of information organization, also meant the beginning of the information retrieval and information services.

Integration was the optimization of heterogeneous normative and non normative resources combination, and the creative process of integration and reorganization of resources. Its purpose was to the overall improvement, structure and function of the integrated system, enabled the system to produce digital resources to maximize the function and efficiency. Digital resources integration has the automation, content integration and technical features diversification. As a comprehensive and systematic project, in the implementation of the digital resources integration should follow the principles of integrity, continuity, hierarchy, targeted.

\section{Clouding Computing Technology}

Through the Internet, cloud computing provided the dynamic, expandable and virtualization of resources, was the increase of the related services based on Internet, use and delivery mode [4]. Now the widely accepted was the definition of National Institute of Standards and Technology from the USA. The cloud computing was a kind of pay by usage pattern, this pattern provided the available, convenient, on-demand network access. Entered the configurable computing resources shared pool (resources including network, servers, storage, applications, services). These resources could be quick to provide, just in the management of the very few and or little interaction with service providers. Using cloud computing technology was not only a simple collection of all kinds of resources, but also provided us with a convenient management mechanism, and developers would have transparent freedom of access to resources and the use of resources. Cloud computing provided super computing power for the network application, through certain coordinate scheduling, the cloud computing could provide tens of thousands or even millions of joint between the normal computers to provide a strong.

$94 \quad$ Journal of Information Security Research Volume 10 Number 3 September 2019


In the "cloud" service mode, the integration of resources and scheduling would take the user's needs as the starting point and the foothold, with dynamic adaptability to aggregate information resources and services system, the entire guide information, tracking user behavior. Through the use of cloud computing methods and techniques to create different channels, different sources and different carrier of the integration of resources and the polymerization process to form new knowledge, to realize the connotation of knowledge service deepening, support knowledge retrieval, and took the initiative to push service. Cloud computing environment of digital resources integration was to realize from the traditional normative to dynamic, non normative network resources polymerization of change; From the polymerization to restructuring and integration of information resources. The creative concept of cloud computing service mode and technology, for the digital resources integration and service innovation towards cloud expand the new space for development. Compared with traditional computing, cloud computing key features:

\subsection{Dynamic Resource Allocation}

System dynamic allocation or release different virtual or physical resource depended entirely on the user's demand, when demand increases, the quick matching available resources, to provide users with flexible resources; When users no longer needed the resource, these resources could be quick released, Cloud computing was to provide users with unlimited use of IT resources, and realize the scalability of resources. Cloud computing was to provide users with unlimited use of IT resources, and realize the scalability of resources.

\subsection{Self-support Service Demand}

Cloud computing resources services for the users with the method of self-support, users did not need to interact with suppliers, through the cloud computing system to provide users with a certain application service directory, the user can according to their own needs. Choose a certain service content or project, took a self-service yourself for computing resources ability, and pay of the cost of purchasing service.

\subsection{Transparency of the Resource Pool}

For cloud service providers, all of the underlying resources (storage, computing, network, logic, etc.) formed a "pool", it could be unified management and scheduling, the boundary of the various resources was broken, heterogeneity were blocked up; For users on the use of resources, no need to understand the internal structure of the system, based on their own requirements, the needed resources was infinite and transparent.

\subsection{Network}

Cloud computing services for users was via the Internet by the user through a variety of terminal equipment and standard application to access the Internet everywhere the cloud resource and cloud services.

\section{Digital Resources Integration under the Environment of Cloud Computing}

According to the integration of different requirements, the digital resources integration had a variety of type, level and way, according to the structure of digital resource and the degree of resource integration, resource integration could be divided into data integration, information integration and knowledge integration of three methods for integration. Could Computing has many features, see below:

\begin{tabular}{|c|c|}
\hline \multirow{6}{*}{ The basic features of cloud computing } & 1. Based on the network \\
\hline & 2. Distribute based on need, Charge based on volume. \\
\hline & 3. Support large amount of information processing \\
\hline & 4. Accept Heterogeneous Business System \\
\hline & 5. Support resources dynamic scaling and flow \\
\hline & 6. Accept heterogeneous resources \\
\hline
\end{tabular}

Chart 1. Cloud computing features 
The data of the logical or physical merge data integration was the lowest level of resource integration system, and integrated to a certain extent, and solved the problems of data sharing and exchanging the heterogeneous system. But the data integration only showed the resources quantity change, there was no connection between the data. Information integration was based on data integration at an intermediate level of resource integration system and the optimization of the digital resources. At the same time, in the amount of data integrated also revealed the connection between data, but there was still no involved in semantics. Based on data integration and information integration of knowledge integration were at the highest level in the resource integration system, focused on the restructuring of the concepts and relationships, became the ultimate goal of resource integration. In the practice of digital resources integration, generally, it was a kind of integrated way, and with the main function of mixing of other ways to integrate; They constituted a complete system of digital resources integration. Data integration, information integration and knowledge integration showed cross overlap each other and step by step a progressive process of the development of digital resource integration.

\section{References}

[1] Chinabyte. IDC's latest digital universe research report shows China's growth is the most significant. [2013-5-10]. http:// m.chinabyte.com/storage/270/12553270_m.shtml

[2] China international network information center. China Internet network development state statistic report. [2014-8-30]. http:/ /www.cnnic.net.cn/hlwfzyj/hlwxzbg/hlwtjbg/201407/t20140721_47437.htm

[3] MA, Wen-feng., DU, Xiao-yong. (2007). Digital resources integration: theory, method and application. $2007,46$.

[4] BIDU. Cloud computing. [2014-8-30]. http://baike.baidu.com/

[5] XIE, Fang. (2014). Study on the function and construction of wisdom library, Library Science Research, 06, $15-20+11$.

[6] WEI, Ping. (2014). The digital library service model based on cloud computing research, Lantai world, 02, 43-44.

[7] LI, Chun-lan., DENG, Zhong-hua. (2014). For the library cloud computing research were reviewed, The Library Magazine, 2014, 05, 68-77.

[8] WANG, Ping. (2013). The application of cloud computing in library resources integration and retrieval, Library Journal. 4 , $115-117$

[9] DENG, Xiao-yan. (2013). Construction of digital library based on cloud computing concept, its background of digital library project, The Library Study, 05, 93-96.

[10] Sheng-CHANG, (2013). Cloud computing database query algorithm in the application of the library digital services, Digital Technology and Application, (05) 156.

[11] YIN, Hong., LIU, Wei. (2013). A new generation of library service system: function evaluation and vision, Journal of Chinese Library, 05, 26-33.

[12] LI, Xiao-ming., JIANG, Xiao-yi. (2013). Cloud computing and influence to library's basic framework of business research, Library Construction, 10, 56-60. 